\title{
LA FOBOLOGIA, ¿CIENCIA O FORMA DE ENTRETENIMIENTO?
}

\author{
Maximiliano E. Korstanje \\ Universidad de Palermo, Argentina
}

Resumen.- En los últimos años, el miedo se ha transformado un tema de conversación ya sea por la proliferación de desastres naturales, el crimen urbano, la guerra contra terror, teorías apocalípticas, o estudios sobre el riesgo nuclear. Como sea el caso, asistimos al nacimiento de un nuevo milenio con un grado de incertidumbre elevado que hace colapsar nuestro sistema de seguridad ontológica. Dentro de este contexto, el presente artículo ofrece un nuevo punto de vista en el debate de si es necesario o no construir una nueva ciencia que haga del temor y de sus derivadas el riesgo, la angustia o pánico su objeto de estudio. Consideramos, que la fobología es la disciplina científica más apta para tratar este tipo de fenómenos en el futuro.

Palabras clave.- miedo, riesgo, amenaza, seguridad, fobología

\begin{abstract}
In last years, a wide range of manifestations linked to fear have been certainly become in a serious concern and challenge for citizenry worldwide. Ranging from the war on terror, outbreak of a new virus, natural or man-made disasters to the risk of a nuclear accidents we are now witness (in the inception of a new millennium) of a proliferation of fears and panic. As a result of the high-related degree of uncertainty that characterizes our postmodern life of consumption, our ontological state of security seems to be overloaded. As the previous argument given, the present paper provides readers with a new discipline to research these types of issues in a near future, the phobology. To what an extent the phobology can study issued related to fear and derived expression as panic, risk or hazards, is being placed under the lens of scrutiny in this valuable work.
\end{abstract}

Key Words.- fright, risk, threat, security, phobology

\section{Introducción}

Indudablemente, fue luego de la intervención del psiquiatra austriaco Sigmund Freud que el mundo intelectual comenzó a usar el término "fobia" para describir un comportamiento ligado a un miedo intenso y en ocasiones extremo a determinada situación u objeto. No obstante, Freud parece no haber reparado en la dimensión que tenía la palabra. Fobia viene del griego phobos que simboliza no solo un sentimiento expandido de terror sino también un miedo moderado (Freud, 1998). Antiguamente, los griegos lo utilizaban para simbolizar el pánico (phemobai) que despertaba la batalla y la guerra. Desde ese entonces, si bien con diferentes matices, los psicoanalistas no han cuestionados los postulados iniciales del doctor Freud con todo lo bueno y lo malo que ello implica (Saurí, 1984) (Klein, 1987) (Montiel, 1989) (StewartAngras, 1989) (Marks, 1990) (Nardone, 1997) (Méndez-Carrillo, García y Rodríguez, 1997) (Winnicott, 1996) (Dio-Bleichmar, 1991) (Van-Rillaer, 2000) (Pérez-Grande, 2000) (Ward, 2001). Desde una perspectiva clínica, la naturaleza de la fobia se ha consolidado como un temor patológico extremo 
paralizante el cual impide la realización de diferentes aspectos de la vida social del paciente generando serios problemas de relación y de auto-estima.

Sin embargo, como ha afirmado B. Malinowski, el temor es parte de nuestra vida como entes biológicos y una "concomitante funcional" en la estructuración política de las sociedades. Entre las necesidades básicas, la seguridad se ubica en cuarto lugar. En este sentido, la necesidad de seguridad da como resultado que los hombres utilicen diferentes elementos culturales para su propia protección ${ }^{1}$. La función de la protección en las estructuras culturales hace a la defensa del territorio o la organización hacia peligros externos como ser cataclismos naturales, invasiones etc. Para Malinowski, el principio concomitante de protección consiste simplemente en planear y prever con arreglo a procesos más amplios de adiestramiento en donde se prepara a las jóvenes generaciones para hacer frente a las amenazas del medio ambiente de la forma más eficiente posible (Delumeau, 1978) (Malinowski, 1967) (Dupuy, 1999) (Oszlak, 2006) (Douglas, 2007) (Alexander, 2008).

En los últimos años los científicos se han preocupado por la proliferación de eventos naturales de impacto inmediato sobre la producción y la forma de vida de las personas. Desde megahuracanes, inundaciones, incendios, sequías producidas por el llamado "efecto invernadero". A ello se le suman teorías apocalípticas, las cuales pregonan el fin del mundo, y eventos televisivos guiados como un show sobre ataques terroristas, crimen etc. Palabras tales como riesgo, desastre, fin del mundo, angustia, miedo, pánico y terrorismo se han puesto en boca de la opinión pública en todo el mundo. Sin embargo, cada una de ellas significa algo totalmente diferente y sus aplicaciones obedecen a contextos disímiles. En consecuencia, estamos en condiciones de afirmar que las sociedades pueden ser estudiadas por esas construcciones culturales a las cuales denominamos miedos, y dentro de esta coyuntura, la fobo-logía (estudio de los temores) se debe constituir como la disciplina académica especializada en este tipo de estudios. Esta nueva disciplina se encuentra en condiciones de estudiar conceptos complejos tales como de seguridad, miedo, riesgo, amenaza, desastre, angustia, como así también los diferentes usos que las industrias culturales como el cine o el periodismo hacen del temor. El siguiente trabajo es una reflexión profunda sobre las limitaciones y potencialidades de al fonología como nuevo método científico en los albores de un siglo que se presenta como cambiante y caótico.

\section{Definiendo Conceptos}

Todos, en algún momento de nuestras vidas, hemos sentido miedo. El imaginario colectivo toma al miedo como un sentimiento ambivalente que en ocasiones genera placer y en otras, angustia. No obstante, para arribar a una definición científica de miedo debemos adentrarnos en las contribuciones de la psicología ambiental y cognitiva, la cual nos dice, que el miedo es un sentimiento humano básico cuya función es la supervivencia del organismo. Tal vez una de las limitaciones conceptuales más comunes en este tipo de casos,

\footnotetext{
${ }^{1}$ Metabolismo, Reproducción, Bienestar, Seguridad, Movimiento, Crecimiento y Salud
} 
es la cantidad de sentidos que los científicos asignan a los términos (polisémico). El antropólogo mexicano F. Briones-Gamboa sugiere que temor, riesgo y angustia han sido formas humanas y culturales de intelectualizar el peligro a lo largo de los años (Briones-Gamboa, 2007). Por ejemplo, en Aristóteles de Estagira el temor se refería a un sentimiento que nos lleva a evitar el "displacer" (Aristóteles, Ética II). Más tarde con el descubrimiento de América, la sociedad europea conocería una nueva forma lingüística para el miedo de los antiguos, el riesgo (derivado del latín resecum que significa aquello lo cual corta) (Giddens, 1999; 2000). Pero en las líneas sucesivas veremos que los conceptos, aún en lo que comparten, se distancian en forma considerable.

En consecuencia, podemos definir al temor como una emoción básica sujeta a un objeto limitado, distinguible en tiempo y espacio cuya función principal es darle al organismo (animal o humano) una rápida respuesta frente a un peligro dado, como ser la huida o el enfrentamiento (Fraisse, 1973) (Panksepp, 1982) (Levenson, Ekman y Friesen, 1990) (Strongman, 1996) (Miranda-Conde, 1999). Por lo tanto, el miedo no puede ser intelectualizado previamente a su aparición. Por el contrario, para J. Saurí y otros autores, el temor actualiza un proceso profundo de ritualización con el fin de destruir el suspenso y la incertidumbre propia del futuro (Saurí, 1984). No obstante, evidencia sustancial sugiere que el temor se encuentra presente en la mayoría de los seres vivos como una forma segura de aprendizaje (Miranda-Conde, 1999). A diferencia de la angustia, el temor opera en la mayoría de los casos en un tiempo presente y está determinado por una acción concreta la cual pone al sujeto al límite de su seguridad ontológica. En tanto que amenazado, el organismo responde en forma instintiva.

La angustia, en tanto que sentimiento secundario (combinación de temor y ansiedad) obedece a naturaleza "estable" que atraviesa toda la transversalidad en la vida del sujeto. La formación de la angustia se refiere a los "valores individuales" de cada sujeto con arreglo a una respuesta anticipatoria ante una realidad la cual se presenta como difusa e incierta. La angustia no solo se encuentra condicionada por la racionalidad frente a la incertidumbre sino que también representa la posibilidad de libertad frente a la nada existencial (Sierra, Ortega y Zubeidat, 2003) (Strongman, 1996) (Levinas, 2000) (Kierkegaard, 2003; 2006; 2007) (Heidegger, 1996; 1997) (Giddens, 1991) (Zubiri, 1991) (Nardone, 2007). La angustia, siempre anclada en un futuro imaginado por el hombre, posee una doble dinámica. Mientras por un lado hunde de toda relación con un territorio, por el otro, abandona al sujeto en el vacío (Heidegger, 1997). Por último cabe mencionar la angustia no ejerce estimulo directo sobre el hombre, precisamente su malestar radica en la ambigüedad, la incertidumbre y su falta de límites concretos. Es cierto que en algún punto el miedo y la angustia dialogan. Para comprender mejor la diferencia entre una y otra, veamos el siguiente ejemplo. Cuando el segundo avión se estrelló en las Torres Gemelas, miles de neoyorquinos corrían por las calles en busca de refugio. Ellos no conocían los detalles del atentado como tampoco sus efectos, simplemente emprendían una huida para salvar sus vidas (miedo). Por el contrario, cuando días después de conocidos los pormenores y la forma en la cual se había perpetrado el atentado, la sociedad americana comenzó a sentir 
un malestar generalizado con respecto a la posibilidad de un atentado nuclear en suelo estadounidense (angustia). Metodológicamente, uno de los problemas principales con la angustia es su inadecuación para ser operacionalizada en variables mutuamente excluyentes, hecho por el cual la hace un objeto de estudio para la filosofía pero no puede ser utilizada por al Ciencia. En otras palabras, no podemos hablar de grados de angustia ya que ella sólo es una. Por su parte, y en tanto que no puede ser medida, la angustia representa serios problemas para los estudios científicos de campo.

\section{El Riesgo y Las Amenazas}

Definir que se entiende por riesgo puede ser una tarea harto inmensa. En principio, porque cada escuela y sub-disciplina maneja su propia noción de riesgo, segundo porque no existe consenso general sobre que es el riesgo, en tanto que a) categoría cognitivo-perceptiva del sujeto, b) probabilidad de daño futuro o amenaza, c) contexto en el cual se estructuran los peligros del ambiente (sentimiento de inseguridad). Tentativamente, K. Tierney define al riesgo como toda posibilidad de concreción de efectos no deseados los cuales se presentan como ajenos al sujeto y pueden afectar su sentido de seguridad (Tierney, 1994). Existe dentro de las Ciencias Sociales una corriente la cual toma al riesgo partiendo desde el paradigma del daño mientras una segunda corriente estudia la convergencia entre las construcciones socioculturales respecto a ciertos peligros o amenazas, que bien pueden ser imaginarias o reales. Para esta segunda vertiente lo más importante no es el daño-a-futuro en sí, sino comprender como trabajan las elaboraciones simbólicas en cada sociedad. Desde esta perspectiva, es lícito hablar de una "narrativa" o "discurso del Riesgo" (Zinn, 2010).

Slovic y Weber sugieren que existen diferentes acepciones sobre lo que comprendemos por riesgo. Los usos más frecuentes son 1) el riesgo como una amenaza; 2) el riesgo como probabilidad, 3) el riesgo como consecuencia de una decisión y 4) el riesgo como una potencial adversidad. Para los autores, el riesgo es solo un concepto construido por los seres humanos para comprender los peligros, incertidumbres y amenazas de la vida. El problema que se presenta con la evaluación del riesgo es que como constructo subjetivo en ocasiones no es plausible de ser cuantificado. Por ejemplo, un fumador puede ver como riesgoso abordar a un avión mientras subestima los daños potenciales su propia costumbre con respecto al cigarrillo. Uno de los aspectos centrales en los estudios de los riesgos es la amplificación emocional que implica para la sociedad (Slovic y Weber, 2002).

U. Beck ha enfatizado en sus trabajos que la sociedad burguesa cuya característica principal de diferenciación ha sido la pobreza y la riqueza (nivel de producción material) debe ceder paso a la "sociedad del riesgo" en donde los riesgos son alojados y diseminados en todas las "clases sociales" (Beck, 2006). Precisamente, una de las funciones de los riesgos no solo es aumentar el consumo sino desdibujar las fronteras morales entre quienes tienen el poder y quienes lo deben padecer. Para A. Giddens, el riesgo fue inicialmente un concepto acuñado por los exploradores españoles y portugueses luego de la 
Conquista de América para simbolizar las eventualidades que encontraban a su paso las cuales podían hacer fracasar la expedición. Si bien las culturas anteriores a la modernidad tenían un concepto definido del miedo, no hablaban expresamente de riesgo. Este término sólo es posible en una sociedad que se orienta hacia el futuro y trata de olvidar su pasado; la aplicación de la palabra riesgo se encontraba vinculada a la incertidumbre y/o temor que inculcaba en el explorador un espacio desconocido (Giddens, 2000: 53).

Por su parte, Douglas y Wildavsky definen cuatro tipos culturales de personalidad: igualitarios, individualistas, jerárquicos y fatalistas. Cada uno de ellos percibe a los peligros de diferente forma. Por medio de una explicación que combina grados de socialización con arreglo al sentido de pertenencia a un grupo con el apego o internalización de las normas, Douglas y Wildavsky consideran que los jerárquicos poseen un alto grado de socialización y un alto apego a las normas, mientras el igualitario posee un alto grado de socialización pero bajo apego a las normas. Asimismo, mientras el nihilista muestra en bajos grados de socialización y respecto por las normas por igual, el individualista tiene un alto apego por las normas pero un bajo grado de socialización de grupo (Douglas y Wildavsky, 1983). El énfasis en la manera en la cual quienes mantienen el poder transmiten el riesgo se ha denominado "comunicación del riesgo" y atañe la labor de periodistas, científicos, políticos etc (Kahan et al, 2006; 2008). Para algunos, la comunicación del riesgo se ha transformado en un arma de adoctrinamiento político, como el caso del terrorismo, o de simple show cuyo fin es entretener a la audiencia (Baudrillard, 2002)

No obstante, una de las limitaciones de la teoría de la percepción del riesgo, bien analizada por el sociólogo alemán Niklas Luhmann, es la inconsistencia entre lo que representa un riesgo y una amenaza. Todo riesgo se constituye en torno a una utilidad contemplada previamente ya sea como producto de la incertidumbre o de la contingencia, eso quiere decir que debe existir la posibilidad de poder evitar el daño. Los tratamientos actuales del tema, ya sea en Giddens o Beck, no hacen más que promover teorías apocalípticas sin sentido. El riesgo está determinado por un proceso de decisión previa en donde quien se encuentra involucrado conoce los efectos derivados. Por regla general, quien toma las decisiones no es quien debe padecer las consecuencias. La distinción entre riesgo y peligro supone que mientras el primero se considera una consecuencia directa de la decisión (riesgo de decisión) el peligro es externo y atribuible al entorno.

En resumen, mientras el peligro requiere una actitud pasiva, el riesgo sugiere todo lo contrario. La caída de un avión, un terremoto, o un crimen no pueden ser considerados riesgos porque el involucrado no está sujeto al principio de contingencia y no hay nada que pueda hacer para evitar el infortunio (Luhmann, 2006). Ha sido precisamente, esta observación aquella por la cual la mayoría de los estudios vinculados al riesgo colapsan sobre todo en el mundo anglosajón en donde no existe la diferencia conceptual entre "risk and threat". En consecuencia, la fonología como nueva ciencia se ocuparía de todos los conceptos mencionados, y sus aplicaciones prácticas a las cuestiones diarias de las personas. 


\section{La Cuestión del Método ¿qué estudia la fobo-logía?}

Existen actualmente una cantidad abismal de estudios cuantitativos, bajo modelo multivariado y provistos de algoritmos matemáticos orientados a estudiar problemas ligados a la percepción del riesgo o los peligros (Somnez, 1998) (Graham y Clemente, 1996) (Hunter, 2006) (Hoxter-Lee y Lester, 1988) (Chauhan, 2007) (Burns, 2007). No obstante, lo que estos trabajos no pueden captar es la relación significante entre dos o más variables. El modelo multivariado explica muy bien que las mujeres perciben los riesgos o los peligros con mayor intensidad con respecto a los hombres, sin embargo, los modelos cuantitativos poco pueden decir (a pesar de su gran extensión de muestra) sobre las causas subyacentes de esa relación. En otras palabras, estos trabajos desconocen la relación de rol entre hombres y mujeres y su influencia en la percepción de los peligros. Asimismo, mientras la mujer es socializada para admitir abiertamente sus emociones, el hombre por lo general confunde temor con ira (Hotchschild, 1979; 1983) (Strongman, 1996) (Kessler, 2009).

R. Stalling llama la atención sobre la necesidad de estudiar sociológicamente a riesgos y amenazas. Ambos conceptos involucran personas que reaccionan ante eventos similares que se dieron en el pasado sobre ellos o sobre su comunidad con el único fin de evitar que se produzcan en el futuro. La esencia de la rutinización estructural de la sociedad abre la posibilidad de ser vulnerable ante posibles disrupciones. En este contexto, Stalling afirma que la vulnerabilidad del Estado Nación como tal se constituye en el proceso de legitimación cuya máxima expresión es la política, y en segundo grado el mercado por medio de la economía (Stalling, 1997).

Fue así que el mercado ha hecho de la fobología una forma de entretenimiento la cual se encuentra presente en todos los hogares 24 horas al día por medio de películas, noticieros, documentales etc. El temor ha sido mercantilizado en un show cuya función conlleva inevitablemente al adoctrinamiento interno (control social) (Altheide, 2006; 2009) (Sontag, 2002) (Said, 2001) (Holloway and Pelaez, 2002) (Bernstein, 2006) (Baudrillard, 1995a; 1995b; 2006) (Gray, 2007) (Smaw, 2008) (Corey, 2009) (Wolin, 2010) (Howie, 2009) (Skoll, 2007) (Smaw, 2008). Éste tema ha sido ampliamente estudiado por los postmodernos franceses como el caso de J. Baudrillard quien directamente llamó al World Trade Center el "Espectáculo del Desastre". Su tesis principal es que el accidente ha dado paso no solo a la manipulación de los Mass-Media sino a la industria del entretenimiento. De hecho, los noticieros y periódicos se han convertido en una forma cínica de entretenimiento en donde se prioriza la inseguridad/ o sufrimiento del otro con la comodidad del hogar (Baudrillard, 1995a, 1995b, 2006) (Virilio, 2007). Los viajes y la industria del turismo se han sensibilizado con los efectos del World Trade Center y la lucha contra el terrorismo (Weber, 1998) (Domínguez, Burguette and Bernard, 2003) (Kuto and Groves, 2004) (Aziz, 1995) (Castaño, 2005) (Robson, 2008) (McCartney, 2008) (Floyd and Pennington-Gray, 2004) (Paraskevas and Arendell, 2007) (Sackett y Botterill, 2006) (Essner, 2003) (Kozak, Crotts and Law, 2007) (Yuan, 2005); pero lejos de escudriñar sobre las causas y la conexión entre ambos, se han 
perfilado por minimizar las consecuencias negativas sobre el consumo e industria, dejando a un lado la vocación científica por el saber. El recuerdo de un momento doloroso se deifica a través de los medios de comunicación en la creación de un discurso político incuestionable y hegemónico cuya función es desdibujar las reales causas del desastre (Quarantelli y Wenger, 1989) (Quarantelli, 2006) (Rodríguez, Díaz y Aguirre, 2004) (Zizek, 2009) (Korstanje, 2010; 2011).

\section{Conclusión}

Tentativamente puede definirse a la fobología como aquella disciplina cuyo eje de interés es el estudio del miedo en todas sus formas. Por lo pronto, si bien una gran cantidad de trabajos y bibliografía seria puede ser recomendada en cuanto a riesgo, teorías apocalípticas y desastres reales. Por un tema de tiempo y espacio, el siguiente ensayo ha sido un humilde intento por recalcar la necesidad de una nueva disciplina científica capaz de amalgamar todos los aspectos que hacen al temor como sentimiento humano y objeto científico a la vez que explique la conexión entre las diferencias culturales y las formas en que las sociedades elaboran, intelectualizan y digieren la incertidumbre (Dupuy, 1999). En ese contexto, la fobo-logía abarca los siguientes tópicos: a) seguridad (Castel, 2006) (Bauman, 2008) (Beck, 2006) (Foucault, 2001; 2006), b) miedo (Corey, 2009), c) angustia (Heidegger, 1996) (Kierkegaard, 2003; 2006; 2007), d) riesgo (Beck, 2006) (Gough, 2000) (Giddens, 1991) (Luhmann, 2006), e) entretenimiento cultural (Virilio, 1991; 2007), f) amenazas, g) desastres naturales o provocados por el hombre (Fritz y Mathewson, 1957) (Erikson, 1994) (Nigg, 1995) (Dynes, 1998; 1999) (Fielding et al, 2005) (Zolala, 2010) (Quarantelli, 2006) (Korstanje, 2011) (Paton y Johnston, 2009) (CastroGarcia, 2006) (Connell, 2001) (Skoufias, 2003) (Crowards, 2000) (Dalhammer y Tierney, 1996) (Aguirre y Quarantelli, 2008) (Haigh y Amaratunga, 2010) (Drury, Cocking y Reicher, 2009), h) temas clínicos de ansiedad vinculados a ataques de pánico, fobias y miedos en general, (Saurí, 1984) (Klein, 1987) (Montiel, 1989) (Stewart-Angras, 1989) (Marks, 1990) (Nardone, 1997) (Méndez-Carrillo, García y Rodríguez, 1997) (Winnicott, 1996) (Dio-Bleichmar, 1991) (Van-Rillaer, 2000) (Pérez-Grande, 2000) (Ward, 2001), i) terrorismo y medios masivos de comunicación (Baudrillard, 2002; 2006) (Essner, 2003) (Bernstein, 2006) (Corey, 2009) (Fromm, 2005), j) teorías religiosas y seculares apocalípticas y k) procesos de victimización y temor al crimen (Flier, 1999) (Kessler, 2009) (Entel, 2007) (Miguez e Isla, 2010). Todo éste bagaje amplio de temas tiene exactamente el mismo origen funcional que brillantemente ha estudiado B. Malinowski: el principio de protección en sus tres facetas metodológicas las cuales se conectan, excluyen e incluyen mutuamente:

1) Faceta Biográfica: corresponde a la narrativa intenta de cómo representa el individuo el temor ante un evento no esperado como así también las diferentes simbolizaciones que el sujeto hace frente al principio de incertidumbre.

2) Faceta Grupal. Se refiere a la construcción de un complejo proceso antropomórfico de reificación en donde los temores del sujeto se negocian, equilibran o potencian con respecto a un-otro. Mi miedo, puede desvanecerse, 
desaparecer o reafirmarse frente al miedo del otro. Por ejemplo, el crimen urbano representa un fiel reflejo de cómo esta faceta opera a nivel grupal.

3) Faceta Societaria. En a cual intervienen 4 subsistemas que fundan toda sociedad, el sistema político, económico, mítico-religioso y onírico. Éste último es de capital importancia debido a que regula las incongruencias en la interacción de los otros tres subsistemas restantes. El sistema onírico comprende al ocio, el entretenimiento, el turismo, y otras formas de evasión física y psicológica. Como su nombre lo índica el sistema onírico tiene dos funciones, la primera es poner temporalmente el sujeto fuera del ámbito normativo para su restitución mental y física; pero a la vez, su función más importante es revitalizar todos los tendones sociales que hacen del hecho social su razón de ser. Por ejemplo, los valores culturales.

La fobología debe operar tanto en forma biográfica como grupal y societaria alternando métodos cuantitativos como los cuestionarios estandarizados, las estadísticas, los programas de emulación, técnicas de correlación y regresión etc, como también los cualitativos observación participante y no participante, análisis de contenido, visual y por supuesto la etnografía. Sin embargo, a diferencia de las Ciencias Sociales en general como la psicología, antropología y sociología que ya hacen uso de estos métodos, la fobología debe profundizar en los procesos de introspección que históricamente han sido olvidados por el positivismo. La fobología, y con ello pretendo dar un cierre a mi trabajo, debe comprender el complejo, disperso y fragmentado mundo de phobos y su acción sobre el sistema social total. En consecuencia, la fobología es parte integrante de las Ciencias Sociales. El miedo es parte inherente a nuestra vida individual y social.

\section{Bibliografía}

Aguirre, B. E and Quarantelli, E. H. (2008). "Phenomenology of Death Counts in Disasters: the invisible dead in the 9/11 WTC attack". International Journal of Mass Emergencies and Disasters. Vol. 26 (1): 19-39.

Alexander, C. L. (2008). "Safety, Fear and Belonging: the everyday realities of Civic Identity. Formation in Fenham, Newscastel upon tyne". Acme: an International E Journal of Critical Geographies. British Columbia University, Canada. Vol. 7 (2): 173-198. Material disponible en http://www.acmejournal.org/vol7/CLA.pdf. Extraído el 16 de Agosto de 2009.

Altheide, D. (2009). "Moral Panic, From sociological concept to public discourse". Crime and Media Culture. Vol. 5 (1): 79-99.

Aristóteles, de Estagira. (1997). Ética Nicomaquea. México, Editorial Porrúa.

Aziz, H. (1995). "Understanding attacks on tourists in Egypt". Tourist Management, Vol. 16: 91-95.

Baudrillard, J. (1995a). The systems of the objects. Mexico, Siglo XXI. 
Baudrillard, J. (1995b) The Gulf War Did Not Take Place. Sydney, Power Publications

Baudrillard, J. (2002). "La Violence du Mondial". In Power Inferno. Paris, Galilee. Pp. 63-83. Translated to English at www.ctheory.net/text_file.

Baudrillard, J. (2006). "Virtuality and Events: the hell of power". Baudrillard Studies. Vol. 3 (2). July. Availabe at http://www.ubishops.ca/BaudrillardStudies/. Bishop's University, Canada. Version translated by Chris Turner.

Bauman, Z. (2008). Miedo Líquido: la sociedad contemporánea y sus miedos líquidos. Buenos Aires, Paidos.

Beck, U. (2006). La Sociedad del Riesgo: hacia una nueva modernidad. Buenos Aires, Paidos.

Bernstein, R. (2006). The Abuse of Evil. The corruption of politics and religion since 9/11. Buenos Aires, Katz.

Burns, W. J. (2007). "Risk Perception: a review". CREATE REPORT. Center of Risk and Economics Analysis of Terrorism Events. Under office of Naval Research. Report 07-001. University of Southern California. Material disponible en http://create.usc.edu/research/54570.pdf. Extraído el 10 de Octubre de 2009.

Castaño, J. M. (2005). Psicología Social de los Viajes y el Turismo. Madrid, Thomson Ed.

Castel, R. (2006). La Inseguridad social: ¿Qué es estar protegido?. Buenos Aires, El Manantial.

Castro-Garcia, C. (2007). "Imbalances of Genre in integral planning of disaster prevention: an approach". Journal of Cristobal Colon's University. Issue 20 (3): 21-45.

Chauhan, V. (2007). "Safety and Insecurity: Perception of tourists visiting Kashmir, India". Advances in Hospitality and Leisure. Vol. 3: 3-17.

Connell, R. (2001). "Collective Behavior in the September 11, 2001. Evacuation of The World Trade Center". Preliminary Paper \# 313. Disaster Research Center, University of Delaware, United States.

Corey, R. (2009). Fear, the history of Political Ideas. Mexico, Fondo de Cultura Económica.

Crowards, T. (2000). "Comparative Vulnerability to natural disasters in the Caribbean". Caribbean Development Bank Research, Paper 1/00. 
Dalhammer, J. and Tierney, K. (1996). "Rebounding from Disruptive Events: Business Recovery Following the Northridge Earthquake". Annual Meeting of the North Central Sociological Association, Cincinnati, Ohio. April.

Dio-Bleichmar, E. (1991). Temores y Fobias. condiciones de génesis en la infancia. Barcelona, Editorial Gedisa.

Domínguez, P, Burguette, E and Bernard, A. (2003). "Efectos del 11 de Septiembre en la hotelería Mexicana: reflexión sobre la mono-dependencia turística". Estudios y Perspectivas en Turismo. Vol. 12 (3-4): 335-348.

Douglas, M. (2007). Pureza y Peligro: un análisis de los conceptos de contaminación y tabú. Buenos Aires, Nueva Visión.

Douglas, M. y Wildavsky, A. (1983). Risk and Culture: an essay on the selection of technological and environmental Dangers. Los Angeles, University of Californa Press.

Drury, J; Cocking C and Reicher, S. (2009). "The Nature of Collective Reisilience: Survivor Reactions to the 2005 London Bombings". International Journal of Mass-Emergencies and Disasters. Vol. 27 (1): 66-95.

Dulmeau, J. (1978). El Miedo En Occidente. Paris, Fayard Ed.

Dupuy, J. M. (1999). El Pánico. Barcelona, Gedisa Editorial.

Dynes, R. (1998). "Sesimic Waves in Intelectual Currents: the uses of the Lisbon Earthquake in 18th Century thought". Preliminary Paper \# 272. Disaster Research Center, Universidad de Delaware, Estados Unidos.

Dynes, R. (1999). "The Dialogue between Voltaire and Rousseau on the Lisbon Earthquake: the emergence of social Science View". Preliminary Paper \# 293. Disaster Research Center, Universidad de Delaware, Estados Unidos.

Entel, A. (2007). La Ciudad y sus Miedos: la pasión restauradora. Buenos Aires, La Crujía Ediciones.

Erikson, K. (1994). A New Species of Trouble: explorations in Disasters, Trauma and Community. Nueva York, Norton and Company.

Essner, J. (2003). "Terrorism's impacto $n$ Tourism: what the industry may learn from Egypt's struggle with al-Gama'a al-Islamiya". Security and Development. IPS 688.

Fielding et al. (2005). "Avian Influenza Risk Perception, Hong Kong". Emerging Infectious Diseases. Vol. 11 (5): 677-682. Material Disponible en 222.dcd.gov/eid. Extraído el 12 de Octubre de 2009.

Floyd, M. and Pennington-Gray, L. (2004). "Profiling Risk: perception of tourist". Annals of Tourism Research. Vol. 31 (4): 1051-1054. 
Flier, A. (1999). “¿Porqué el síndrome del miedo se instaló en la Argentina?. BAE. 05 de Mayo.

Floyd, M. Gibson, H. Pennington-Gray, L and Thapa, B. (2003). "The Effects of Risk Perception on Intention to Travel in the Aftermath of September 11, 2001". Safety and Security in Tourism: relationships, Management and Marketing. Vol. 15 (2).

Foucault, M. (2001). Defender la Sociedad. Buenos Aires, Fondo de Cultura Económica.

Foucault, M. (2006). Seguridad, Territorio, Población. Buenos Aires, Fondo de Cultura Económica.

Fraisse, P. (1973). "Las Emociones". En Motivación, Emoción y Personalidad. P. Fraisse y J. Piaget (compiladores). Buenos Aires, Editorial Paidos. Pp. 106187.

Freud, S. (1996). Obras completas. Presentación autobiográfica inhibición síntoma y angustia. Volumen XX. Buenos Aires, Amorrortu Editores.

Freud. S. (1998) "Análisis de la Fobia en un niño de cinco años". Obras Completas, volumen X. Buenos Aires, Amorrortu Editores.

Fritz, C. E. and Mathewson, J (1957). Convergence Behaviour in Disaster". Study No. 9. Washington D.C, National Academy of Sciences.

Fromm, E. (2005) El Miedo a la libertad. Buenos Aires, Paidos.

Giddens, A. (1991). Modernity and Self-Identity: Self and Society in the Late Modern Age. California, Stanford University Press.

Giddens, A. (1999). Consecuencias de la Modernidad. Madrid, Alianza Editorial.

Gough, J. (2000). "Perceptions of Risk from Natural Hazards in two remote New Zealand communities". The Australasian Journal of Disaster and Trauma Studies. Vol. 2.

Graham, J. D. y Clemente, K. (1996). "Hazards in the News: Who Believes that?". Risk in Perspective. Harvard Center for Risk Analysis. Vol. 4 (4).

Gray, C. S. (2007). "The Implications of Preemptive and Preventive war Doctrines: Reconsideration". Department of the Army, Department of Defense, US Government. Available at http://www.strategicstudiesinstitute. army. mil/Pubs/display.cfm?pubid=789

Haigh, R. and Amaratunga, D. (2010). "An Integrative review of the Built Environment Discipline's role in the Development of society's resilience to 
Disaster". International Journal of Disaster Resilience in the Built Environment. Vol. 1 (1): 11-24.

Heidegger, M. (1996). ¿Qué Es la metafísica?. Buenos Aires, Ediciones Fausto.

Heidegger, M. (1997). El Ser y el Tiempo. Santiago, Editorial Universitaria.

Hochschild, A. (1979). "Emotion Work, feelings rules and social Structures". American Journal of Sociology. Vol 85 (3): 551-575.

Hochschild, A. (1983). The Managed Heart. The Commercialization of Human Feelings". Berkeley, California University Press.

Holloway, J and Paláez, E. (2002). "La guerra de todos los estados contra toda la gente". En Guerra Infinita: hegemonía y terror mundial. Ceceña, A. y Sader, E. Buenos Aires, CLACSO. Pp. 159-166.

Hoxter-Lee, A. y D. Lester. (1988). "Tourist behaviour and personality". Personality and Individual Differences, 9: 177-178.

Howie, L. (2009). "A Role for Business in the War on Terror". Disaster Prevention and Management. Vol. 18 (2): 100-107.

Hunter, D. R. (2006). "Risk Perception among General Aviation Pilots". The International Journal of Aviation Psychology. Vol. 16 (2): 135-144.

Kahan, D. M. et al. (2006). "Book Review: Fear of Democracy, a cultural evaluation of Sunstein on Risk". Harvard Law Review. Vol. 119, N4: 1071-1109.

Kahan, D. M. et al. (2008). "Cultural Cognition of the risk and benefits of nanothecnology". Nature Nanotechnology. Vol 4: 87-90.

Kessler, G. (2009). El Sentimiento de Inseguridad: sociología del temor al delito. Buenos Aires, Siglo XXI.

Kierkegaard, S. (2003). Temor y Temblor. Buenos Aires, Losada.

Kierkegaard, S. (2006). El Concepto de la Angustia. Buenos Aires, Ediciones del Libertador.

Kierkegaard, S. (2007). Diario de Un Seductor. Buenos Aires, Ediciones del Libertador.

Klein, M. (1987). "El Psicoanálisis de niños". En Obras Completas de Melanie Klein. Tomo 2. Buenos Aires, Editorial Paidos.

Korstanje, M. (2009). "Re visiting the risk perception theory in the Context of Travels". ERTR: e-Review of Tourism Research. Vol. 7 (4). A\&M Texas University USA. 
Korstanje, M. (2011). "Swine Flu in Buenos Aires: beyond the principle of Resilience". International Journal of of Disaster Resilience in the Built Enviroment. Volume 2 (1). In press. University of Salford, UK.

Kozak, M, Crotts, J. and Law, R. (2007). "The Impact of the pereption of risk on international Travellers". International Journal of Tourism Research. Vol. 9 (4): 233-242.

Kuto, B. and Groves, J. (2004). "The Effects of Terrorism: evaluating Kenya's tourism Crisis". Pero ¿como definir un acto "terrorista?. E-review of tourism Research. Vol. 2 (4): 88-95.

Levenson, R. W.; Ekman, P. y Friesen, W.W. (1990). "Voluntary facial expression generates emotions-specific nervous system activity". Psychophysiology, vol. 27: 363-384.

Levinas, E. (2000). Ética e Infinito. Madrid, Machado Libros.

Luhmann, N. (2006). Sociología del Riesgo. México, Universidad Iberoamericana.

Malinowski, M. (1967). Una Teoría de la cultura. Buenos Aires, Editorial Sudamericana.

Marks, I. (1990). "Miedos, Fobias y Rituales”. Ediciones Martínez-Roca.

Méndez-Carrillo, F. Garcia, J. y Rodríguez, J. (1997). "Miedos y Fobias Infantiles". Tratamiento Psicológico de problemas infantiles. JiménezHernández, M (Editor). Pp. 119-132.

Míguez, D. e Isla, A. (2010). Entre La Inseguridad y el Temor. Instantáneas de la Sociedad Actual. Buenos Aires, Paidos.

Miranda-Conde, M. E. (1999). La Experiencia de ver Televisión, respuesta emocional a secuencias audiovisuales de miedo. Tesis Doctoral al cuidado de E. Torres-Lana. Universidad de la Laguna, España.

Montiel, R. (1989). "Miedos y Fobias en la Infancia: revisión de estrategias de tratamiento y abordaje terápeutico desde una perspectiva conductual". Psiquis. Revista de psiquiatría, psicología médica y psicosomática. Vol. 10 (10). 34-44.

Nardone, G. (1997). "Miedo, Pánico y Fobias: la terapia breve". Barcelona, Editorial Herder.

Nigg, J. M. (1995). "Disaster Recovery as a Social Process". Preliminary Paper 219. Quarantelli Resource Collection, University of Delaware, United States.

Panksepp, J. (1982). "Toward a General psychobiological theory of Emotions". The Behavioural and Brain Sciences. Vol. 5 (407-467). 
Paraskevas, A. and Arendell, B. (2007). "A strategic Framework for terrorism prevention and mitigation in tourism destination". Tourism Management. Vol. 28: $1560-1573$.

Paton, D y Johnston, D. (2006). Disaster Reisilience. An integrated Approach. Springfield, Thomas Book.

Pérez-Grande, M. (2000). "El Miedo y sus Trastornos en la Infancia: prevención e intervención educativa". Aula, revista de Pedagogía de la Universidad de Salamanca. Vol. 12: 123-144.

Quarantelli, E. L. (2006). "The disasters of the $21^{\text {st }}$ century: a mixture of new, old, and mixed types". Disaster Research Center, Preliminary Paper 353. University of Delaware, United States

Quarantelli, E. L y Wenger, D. (1989). "A cross Societal comparison of Disaster News Reporting In Japan and the United States". Disaster Research Center, Preliminary Paper 142. University of Delaware, United States

Robson, L. (2005). "Risk Management for meetings and Events". Annals of Tourism Research. Vol. 35 (3): 840-842.

Rodríguez, H; Díaz, W. and Aguirre, B. (2004). "Comunicating Risk and warnings: an integrated and interdisciplinary Research Approach". Disaster Research Center, Preliminary Paper 337. University of Delaware, United States

Said, E. (2001). "The Clash of Ignorance". The Nation. October 4. Available at http://www.thenation.com/article/clash-ignorance.

Saurí, J. (1984). Las fobias. Buenos Aires, Ediciones Nueva Visión.

Sierra, J.C; Ortega, V. y Zubeidat, I. (2003). "Ansiedad, angustia y estrés". Tres conceptos a diferenciar". Revista Mal-estar y subjetividade. Vol. 3 (1): 10-59.

Slovic, P. y Weber, E. (2002). "Perception Risk posed by Extreme Events". Conference. Risk Management Strategies in an Uncertain World. Palisades, Nueva York. Abril 13-12.

Skoll, G. (2007). "Meaning of Terrorism". International Journal for The Semiotics of Law. Vol. 20: 107-127.

Skoufias, E. (2003). "Economic Crises and Natural Disasters, copying strategies and policy Implications". World Development. Vol. 31 (7): 1087-1102.

Smaw, E. (2008). "From Chaos to Contrarianism: Hobbes, Pojman, and the Case of World Government". Essays in Philosophy. Vol. 9 (2): 4-18.

Sontag, S. (2002). "Seamos Realistas". In El Mundo Después del 11 de Septiembre de 2001. (Compilación). Barcelona, Editorial Península. Pp. 59-61. 
Somnez, S. (1998). "Tourism, Terrorism, and political inestability". Annals of Tourism Research. Vol. 25: 416-456.

Stalling, R. (1997). "Sociological Theories and Disaster Studies". Disaster Research Center, Preliminary Paper 249.

Stewart-Angras, W. (1989). "Pánico, como superar los miedos, las fobias y la ansiedad". Barcelona, Labor Ed.

Strongman, K. T. (1996). The Phsychology of Emotions: theories of emotions in perspective. Chichester, Willey and Sons.

Tierney, K. (1994). "Sociology's Unique Contributions to the Study of Risk". Disaster Research Center, Preliminary Paper, 204.

Van-Rillaer, J. (2000). "Miedos, Angustias y Fobias". Madrid, Gaia.

Virilio. P. (1991). La Inseguridad del Territorio. Buenos Aires, La Marca.

Virilio, P. (2007). La Ciudad Pánico: el afuera comienza aquí. Buenos Aires, Libros El Zorzal.

Ward, I. (2001). Las Fobias. Buenos Aires, Ed. Longseller.

Weber, S. (1998). "War, terrorism and tourism". Annals of tourism Research. Vol. 25 (3): 760-763.

Winnicott, D. (1996). El Hogar, nuestro punto de partida. Buenos Aires, Paidos. Zinn, J. O. (2010). "Biography, Risk and Uncertainty - is there common ground for bigraphical Research and Risk Research". FQS, Forum, Qualitative Social Research. Vol. 11 (1). Paper 15. Disponible en http://www.qualitativeresearch.net/index.php/fqs/article/view/1512. Extraido el 01 de Febrero de 2011.

Yuan, M. (2005). "After September 11: determining its Impacts on Rural Canadians travel to U.S". E-review of tourism Research. Vol. 3 (5): 103-108.

Zubiri, X. (1991). "Las Fuentes espirituales de la angustia y de la esperanza". Revista de Filosofía, Vol. 6: 239-245, Universidad Complutense de Madrid.

Zolala, F. (2010). "Data Collection alter massive natural disasters (focusing on Bam earthquake, Iran". Disaster Prevention and Management. Vol. 19 (5): 541547 